\title{
The WEGA Stellarator: Results and Prospects
}

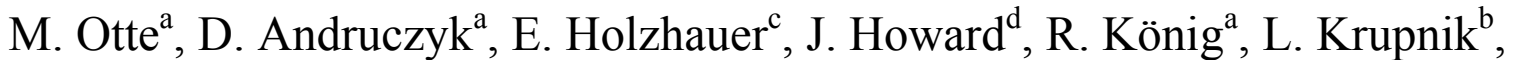 \\ H.P. Laqua ${ }^{a}$, O. Lischtschenko ${ }^{a}$, S. Marsen ${ }^{a}$, J. Schacht ${ }^{a}$, J. Urban ${ }^{\mathrm{e}}$, Y.Y. Podoba ${ }^{\mathrm{a}}$, \\ J. Preinhalter ${ }^{\mathrm{e}}$, F. Wagner ${ }^{\mathrm{a}}$, G.B. Warr ${ }^{\mathrm{a}}$, A. Zhezhera ${ }^{\mathrm{b}}$ \\ ${ }^{a}$ Max-Planck-Institut für Plasmaphysik, EURATOM Association, TI Greifswald, D-17491 Greifswald, Germany \\ ${ }^{b}$ Kharkov Institute of Plasma Physics, Kharkov, Ukraine \\ ${ }^{c}$ Institut für Plasmaforschung, Universität Stuttgart, Pfaffenwaldring 31, D-70569 Stuttgart, Germany \\ ${ }^{d}$ Plasma Research Laboratory, Research School of Physical Sciences and Engineering, ANU, Canberra, ACT 0200, \\ Australia \\ ${ }^{e}$ Institute of Plasma Physics, EURATOM/IPP.CR Association, 18221 Prague, Czech Republic
}

\begin{abstract}
In this article an overview is given on results from magnetic flux surface measurements, applied ECR heating scenarios for $2.45 \mathrm{GHz}$ and $28 \mathrm{GHz}$, fluctuation and transport studies and plasma edge biasing experiments performed in the WEGA stellarator. Examples for the development of new diagnostics and the machine control system are given that will be used at Wendelstein 7-X stellarator, which is currently under construction in Greifswald.
\end{abstract}

Keywords: Stellarator, WEGA, magnetic configuration, ECRH, fluctuation, diagnostic development, Wendelstein 7-X

PACS: $52.35 . \mathrm{Hr}, 52.35 . \mathrm{Ra}, 52.50 . \mathrm{Sw}, 52.55 . \mathrm{Hc}, 52.70 . \mathrm{Ds}, 52.70 . \mathrm{Gw}, 52.70 . \mathrm{Kz}, 52.70 . \mathrm{Nc}$

\section{INTRODUCTION}

WEGA is a medium sized classical stellarator operated at the Max-Planck-Institut für Plasmaphysik, Greifswald branch, since 2001. The primary objectives of WEGA are an educational aspect, i.e. training of students and staff. Furthermore, WEGA is used for basic plasma research and is also test bed for diagnostics that will be installed later on Wendelstein 7-X (W7-X) currently being under construction in Greifswald. WEGA will also be used for testing the prototype of the control system for W7-X. Examples of these aspects are discussed in this article.



FIGURE 1. The WEGA stellarator located at the Max-Planck-Institut für Plasmaphysik, Greifswald branch, Germany. 
Going back in history WEGA is probably one of the longest living experiments in fusion research. Originally WEGA was designed as a hybrid experiment for the study of lower hybrid heating scenarios in a tokamak as well as in a stellarator configuration. In 1975 the machine entered service within the framework of collaboration between France and Germany in Grenoble/France in the tokamak configuration where it was nearly exclusively used in this configuration. Later in the 1980's the machine was operated at the Institut für Physik at University of Stuttgart as stellarator. In 2000 the machine came to the Greifswald branch of the Max-Planck-Institut für Plasmaphysik and was rebuilt in the stellarator configuration. The first stellarator plasma in Greifswald was generated on 13 July 2001.

The plasma vessel of the stellarator version has a circular cross-section with a major radius of $R=72 \mathrm{~cm}$ and a minor radius of $r=19 \mathrm{~cm}$. The maximum averaged plasma radius is $a=11 \mathrm{~cm}$. The plasmas are generated and heated using electron cyclotron resonance heating (ECRH). Two magnetrons with a power of $20+6 \mathrm{~kW}$ each operating at a frequency of $2.45 \mathrm{GHz}$ are used. Typical plasma parameters are in the order of $T_{\mathrm{e}}=5-15 \mathrm{eV}$ and $n_{\mathrm{e}}=10^{17}-10^{18} \mathrm{~m}^{-3}[1]$. In the year 2006 a $28 \mathrm{GHz}$ gyrotron was installed with a maximum power of $10 \mathrm{~kW}(\mathrm{cw})$. Furthermore, the machine is equipped with a 5-period ohmic transformer, which has not been used for plasma heating so far.

\section{MAGNETIC PROPERTIES}

Stellarators generate their confining magnetic field already without plasma, therefore the quality and the structure of the confining field can be measured precisely.

WEGA has a very flexible magnetic configuration. The magnetic field coil system consists of 40 toroidal field coils, 4 helical field coils and 2 pairs of vertical field coils. The helix has a poloidal period number $l=2$ and toroidal period number $m=5$ configuration. Furthermore, an error field compensation coil was installed. In combination with the vertical field coils (VFC) and error field compensation coils (EFCC) WEGA has a very flexible magnetic configuration. The machine can be operated steady state up to a field strength of $B=0.35 \mathrm{~T}$. In pulsed operation maximum field strength of $0.9 \mathrm{~T}$ can be achieved.

\section{Magnetic Flux Surface Measurements}

In order to check the existence and quality of the magnetic flux surfaces the well-known fluorescent electron beam technique was applied [2]. The method bases on the interaction of an electron beam accelerated parallel to the magnetic field vector and a detector coated with fluorescent material. When the electron beam hits the detector, light will be emitted that can be integrated by a camera. Usually, the detector is a moveable rod that allows a scanning of the cross-section or a fixed mesh is installed inside the plasma vessel.

For the mapping of the electron beam a wire ellipse coated with fluorescent $\mathrm{ZnO}$ powder could be moved over the whole vessel cross section resulting in a Poincaré plot. The experiments were performed at two toroidal positions $\left(\varphi=72^{\circ}\right.$ and $\left.144^{\circ}\right)$. For observation a CCD camera was used looking nearly perpendicularly onto the fluorescent detector system. The electron gun was installed at $\varphi=252^{\circ}$ on tip of a manipulator that allowed a radial positioning of the gun. The emitted electron beam had a intensity of about $i_{\mathrm{emm}}=10 \mu \mathrm{A}$ and an energy of $U=300 \mathrm{~V}$ in maximum.

The experiments were carried out scanning a wide range of rotational transform $0.1 \leq \mathrm{l} / 2 \pi \leq 1$ by changing the current in the helical field coils at constant toroidal field $B_{0}=87.5 \mathrm{mT}$. It was found that closed and nested magnetic flux surfaces of good quality exist up to $1 / 2 \pi=1$. However, field errors, probably due to a misalignment between the helical and the toroidal field coils, give rise to magnetic islands with a toroidal mode number of $m=1$ and $m=2$. An example is shown in Fig. 2 were the magnetic flux surfaces for $1 / 2 \pi=1 / 3$ are disturbed by non-natural islands caused by these unwanted error fields. The experimental results were verified by results from field line tracing measurements. Two codes, the Gourdon code, which has been applied for various experiments so far and the new w7-code by A. Werner were benchmarked [10]. The calculations of both codes results are in good agreement with the experiments when including stellarator symmetry breaking errors. I was found that a simple horizontal shift of about $3 \mathrm{~mm}$ between the geometric center of the toroidal field coils and the helical field coils produces a magnetic error field that generates magnetic islands of the same amplitude as found in the experiment. The toroidal phase was adjusted such that the magnetic islands in the code simulations are at the same poloidal position as found in the experiments. 


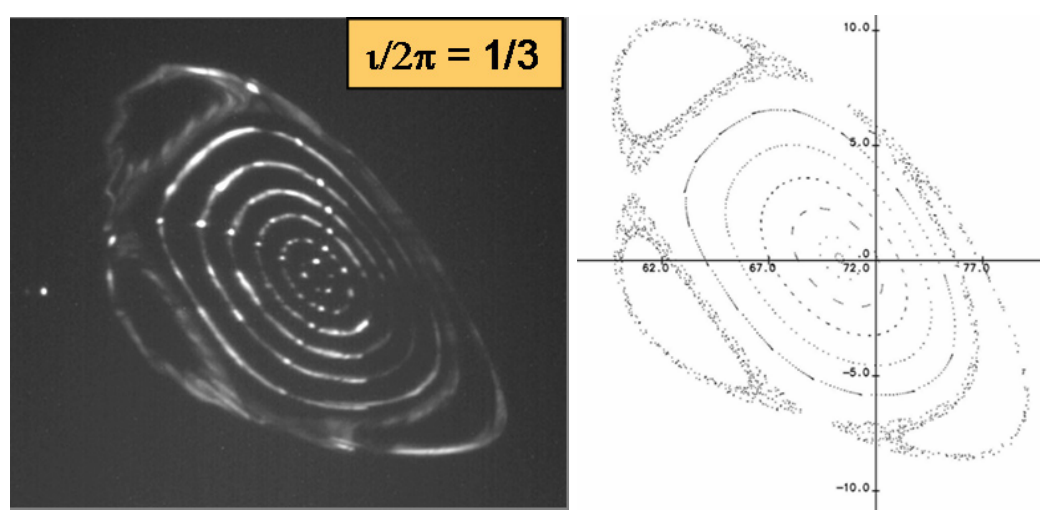

FIGURE 2. Magnetic flux surfaces for a $1 / 2 \pi=1 / 3$ configuration from experiment (left) and field line tracing calculations (right) including a symmetry breaking error field.

In addition to the toroidal and helical field coils WEGA has vertical field coils (VFC) that allow a radial inward or outward shift of the plasma by a few centimeters. The amplitude of the magnetic field that can be generated by the VFCs is typically of the order of $1 \%$ of the toroidal field component only, but shows nevertheless a significant influence on the rotational transform $\mathrm{t}$ by means of increased rotational transform and reduced shear. Thus it is possible to find magnetic configurations avoiding the non-natural and symmetry breaking islands of the order $m=1$ inside the last closed flux surface (lcfs).

In order to avoid these non-natural magnetic islands it is possible to operate WEGA with the VFCs or to manipulate the island size with an additional external error field compensation coil (EFCC). The EFCC consist of a set of up to 4 planar coils with mean diameter of about $34 \mathrm{~cm}$. The coil set is positioned in the horizontal plane in a toroidal position $\varphi=126^{\circ}$ and can effectively compensate the magnetic field errors leading to $m=1$ non-natural magnetic islands as shown in Fig. 3 (left).

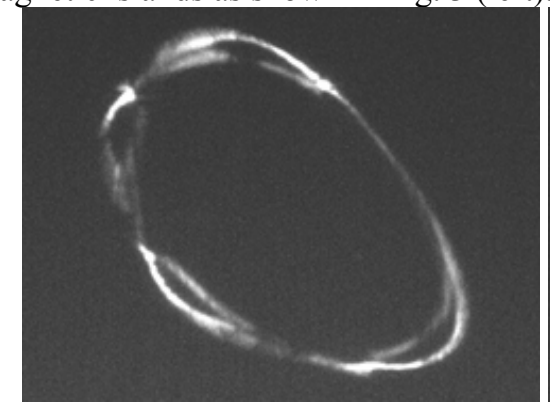

FIGURE 3. Magnetic flux surface for $\mathrm{t} / 2 \pi=1 / 4$
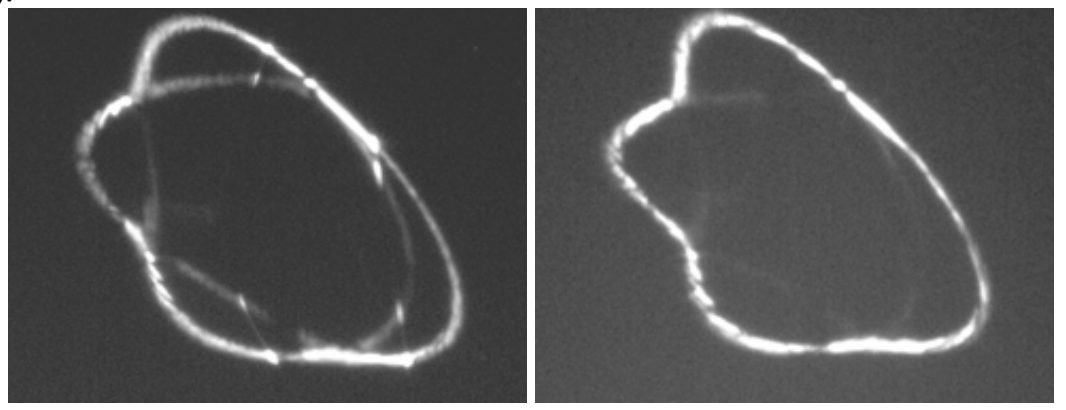

increased islands size (right).

By inverting the field in the EFCC it is also possible to increase the islands size as shown in Fig. 3 (right). While most of the flux surface measurements have been performed for a magnetic field strength of $B_{0}=87 \mathrm{mT}$, the field strength was increased to $B_{0}=0.5 \mathrm{~T}$ in preparation for the experimental campaigns in the future. As a results the existence and quality of the magnetic flux surfaces was conserved and to be more specifically the non-natural islands were also found in same amplitude and poloidal phase. This supports the thesis of a geometrical misalignment of the toroidal and helical field coils, because this error is independent of the field strength.

\section{Visualization of Magnetic Field Lines}

In addition to the flux surface measurements the magnetic field lines have been visualized by means of an electron beam interacting with a background gas. The electrons are emitted by an electron gun with an accelerating voltage of up to $300 \mathrm{~V}$ and a beam intensity of about $100 \mu \mathrm{A}$ and are interacting with the neutrals in the gas. As a result the excited neutrals emit light along the electrons' path of flight. In this sense a magnetic field line can be made visible. At a pressure of $p=5 \times 10^{-5}$ mbar a maximum length of about $65 \mathrm{~m}$ could be detected in hydrogen background gas. A much longer path could be seen during similar experiments in Wendelstein 7-AS where a length 
of $450 \mathrm{~m}$ was visible. It is planned to apply this technique also in Wendelstein $7-\mathrm{X}$ in addition to the magnetic flux surface measurements.

\section{BASIC RESEARCH ON PLASMA WAVE PHYSICS}

\section{Mode Conversion at 2.45GHz ECR Heating}

The plasmas at WEGA are generated and heated by two $2.45 \mathrm{GHz}$ microwave magnetrons with a power of $6 \mathrm{~kW}$ and $20 \mathrm{~kW}$. The antennas are installed at the low field side emitting in ordinary (O) mode. Typically, the plasmas are generated and heated near the resonant magnetic field strength $B_{\text {res }}=87 \mathrm{mT}$ or below allowing discharges with a length of 30 minutes. However the typical length of a discharge is about 1 minute. The electron temperature is in the order of $T_{\mathrm{e}} \approx 10 \mathrm{eV}$, the ion temperature $T_{\mathrm{i}} \approx 2 \mathrm{eV}$. Working gases are $\mathrm{H}_{2}, \mathrm{He}, \mathrm{Ne}$ and Ar. The plasma density is higher than cut-off density of $n_{\text {cutoff }}=7.5 \times 10^{16} \mathrm{~m}^{-3}$, due to a mode conversion [3] from $\mathrm{O}$ into extraordinary (X) mode to Electrostatic Electron Bernstein wave (EBW). The OXB mode conversion process requires launching the HF beam oblique to the magnetic field lines. An optimized $\mathrm{O}$ to $\mathrm{X}$ conversion antenna results in a peaked density profile of $>10$ times higher density than the cut-off density and slightly hollow electron temperature profiles.

The investigation of plasma heating scenario via OXB mode conversion was performed by measurements of the behavior of high frequency waves in the WEGA plasma. A set of HF probes measures the amplitude and phase of the heating wave in the vicinity of the heating antenna. Results of the measurements were compared with simulations that were made using a Finite-Difference Time-Domain (FDTD) code. The measurement results agree well with the full-wave calculations. The observed resonant behavior of the wave between the upper hybrid resonant (UHR) layer and cutoff region shows the conditions for the X-B conversion process are satisfied. The assumption of the over-dense plasma heating by EBW is also supported by Langmuir probe measurements of the plasma parameters with simultaneous heating power modulation. The measurements show the deposition of the HF power deep inside the over dense plasma region [4].

\section{Current drive Experiments}

In cooperation with IPP Prague (Czech Republic) the propagation of the Bernstein waves was calculated for OXB mode conversion at $2.45 \mathrm{GHz}$. Although the antenna has a symmetric emission characteristic in co and counter direction to the magnetic field, the Bernstein waves propagate in a co-direction to the magnetic field. Thus, a toroidal current was predicted. With the help of the primary coils of the ohmic transformer and a Rogowski-coil covering the complete vessel cross section, a current of up to $50 \mathrm{~A}$ could be measured during power modulation experiments. The amplitude of the current did strongly depend on the magnetic configuration and could be inverted by changing the shear. It is planned to expand these experiments by measurements with a mini-Rogowski coil inside the plasma in order to measure the currents locally resolved.

\section{GHz ECR Heating Scenarios}

In cooperation with IPF Stuttgart (Germany) and the CIEMAT (Madrid, Spain) a $28 \mathrm{GHz}$ electron cyclotron resonance heating $(\mathrm{ECRH})$ system has recently been installed on WEGA. This microwave plasma heating system will be used for plasma production and heating studies at a magnetic field strength of $B_{0}=0.5 \mathrm{~T}$. The gyrotron allows steady state operation with an output power of $10 \mathrm{~kW}$. Two heating scenarios are planned. Currently plasmas are produced with second harmonic extraordinary (X2) mode heating. Here, a parabolic mirror is used to focus the Gaussian beam emanating from the end of the waveguide into the centre of the plasma where, using ISS95, $<T_{\mathrm{e}}>=$ $50 \mathrm{eV}$ at $n_{\mathrm{e}}<5 \times 10^{18} \mathrm{~m}^{-3}$ are expected. It is also planned to generate supra-thermal electrons with this heating scenario and compare the results with those from other experiments [11].

Centrally peaked density obtained by Langmuir-probe measurements indicate a central power deposition for onaxis heating $B_{0}=0.5 \mathrm{~T}$. As shown in Fig. 4, off-axis heating experiments resulted in double peaked structure in electron density $n_{\mathrm{e}}$ and temperature $T_{\mathrm{e}}$. In hydrogen discharges the temperatures were higher than $25 \mathrm{eV}$ at the last closed flux surface thus rendering the Langmuir probe diagnostics not useful as it started to emit. Thus this has led the development of ECE and reflectometry diagnostics. By using the curved stainless steel vessel multiple passes of the microwave beam can be achieved. During the experiments a significant drop of the non-absorbed $28 \mathrm{GHz}$ microwave power was measured using a sniffer probe indicating a high absorption efficiency. 
The system will also be used for over-dense OXB mode heating of the plasma. For this a quasi-optical antenna was designed, which provides the best match of the $28 \mathrm{GHz}$ beam to the OXB mode conversion requirements. Here, using ISS95, $<T_{\mathrm{e}}>=25 \mathrm{eV}$ and $n_{\mathrm{e}}>1 \times 10^{19} \mathrm{~m}^{-3}$ are anticipated. This is ideal for wave physics studies and to test W7$\mathrm{X}$ divertor diagnostics.

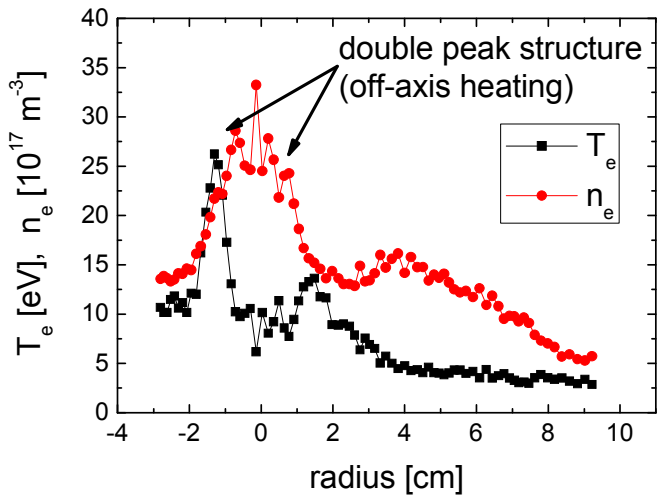

FIGURE 4: Radial profile of electron temperature $T_{\mathrm{e}}$ and density $n_{\mathrm{e}}$ for a hydrogen plasma using $28 \mathrm{GHz}$ gyrotron in X2 mode during off-axis heating.

The system will be used to generate supra-thermal particles for fast particle confinement studies in different magnetic configurations (various $l$ ) in the stellarator geometry. It can also be used for such studies in stellaratortokamak hybrids, when a power supply for the WEGA ohmic heating coils is installed.

\section{BASIC PLASMA RESEARCH ON PLASMA TURBULANCE}

\section{Electrostatic Fluctuations}

In WEGA two probe diagnostics are installed to investigate the spatio-temporal structure of electrostatic fluctuations. A poloidal array, as shown in Fig. 5(left), gives insight into the dynamics of fluctuations perpendicular to $\boldsymbol{B} .13$ equidistant probe tips separated by $d=5 \mathrm{~mm}$ are aligned to the flux surfaces in the edge region. Information about the dynamics of fluctuations parallel to the magnetic field is obtained from measurements, where two probes are placed on the same field line with a distance $L_{\mathrm{c}}$ shorter than the parallel correlation length.

Spectral properties of fluctuations in WEGA are very sensitive to the magnetic field configuration. Depending on rotational transform and toroidal field strength more or less strongly developed turbulence has been found. In case of fully developed turbulence the frequency spectrum is flat up to some $10 \mathrm{kHz}$ and decays following a power law for higher frequencies. Strongest fluctuations are detected in the region of steepest density gradients.

The phase relation between density and potential fluctuations gives information about the underlying instability mechanism. For drift waves a small cross-phase is expected. Since we are dealing with turbulence a whole phase spectrum has to be calculated. In order to measure the cross-phase spectrum, $n_{e}^{\sim}$ and $V_{f l}^{\sim}$ were measured simultaneously by neighboring probes of the array. As expected for drift wave turbulence a cross-phase between density and potential perturbations below $0.25 \pi$ is found on all scales of significant fluctuation power. The finite phase shift indicates the deviation of the plasma from a Boltzmann distribution which is a requirement for instability of drift waves and the development of turbulence.

Applying correlation analysis to data from the poloidal array turbulent structures with a poloidal correlation length of $d_{\mathrm{pol}} \propto 14 \mathrm{~cm}$ and lifetimes of $\tau_{\mathrm{L}} \propto 50 \mu \mathrm{s}$ have been found in $\mathrm{He}$ at $B_{0}=57 \mathrm{mT}$. Structures propagate in electron diamagnetic drift direction with a poloidal velocity of some $\mathrm{km} / \mathrm{s}$.

To investigate the parallel dynamics of turbulence, structures were reconstructed in a poloidal plane. To this end the cross-correlation between data from the reference probe and the array has been calculated. Fig. 3 (right) shows a snapshot of the reconstructed structure for a time lag $\tau=0 \mu \mathrm{s}$. As in the poloidally resolved measurements localized structures with a lifetime of some $10 \mu$ s propagating in $v_{\text {dia,e }}$ have been found.

At $\tau=0 \mu \mathrm{s}$ the center of the structure is displaced against the projection of the reference probe by $d_{\perp} \propto 2 \mathrm{~cm}$. This means, that the observed structure does not propagate along a field line but is slightly tilted against $\boldsymbol{B}$. In wave 
number space this corresponds to a finite parallel wave number with $k_{\|} / k_{\perp}=d_{\perp} / L_{\mathrm{c}} \propto 10^{-2}$. Recent results from the TJ$\mathrm{K}$ torsatron give comparable results [5].
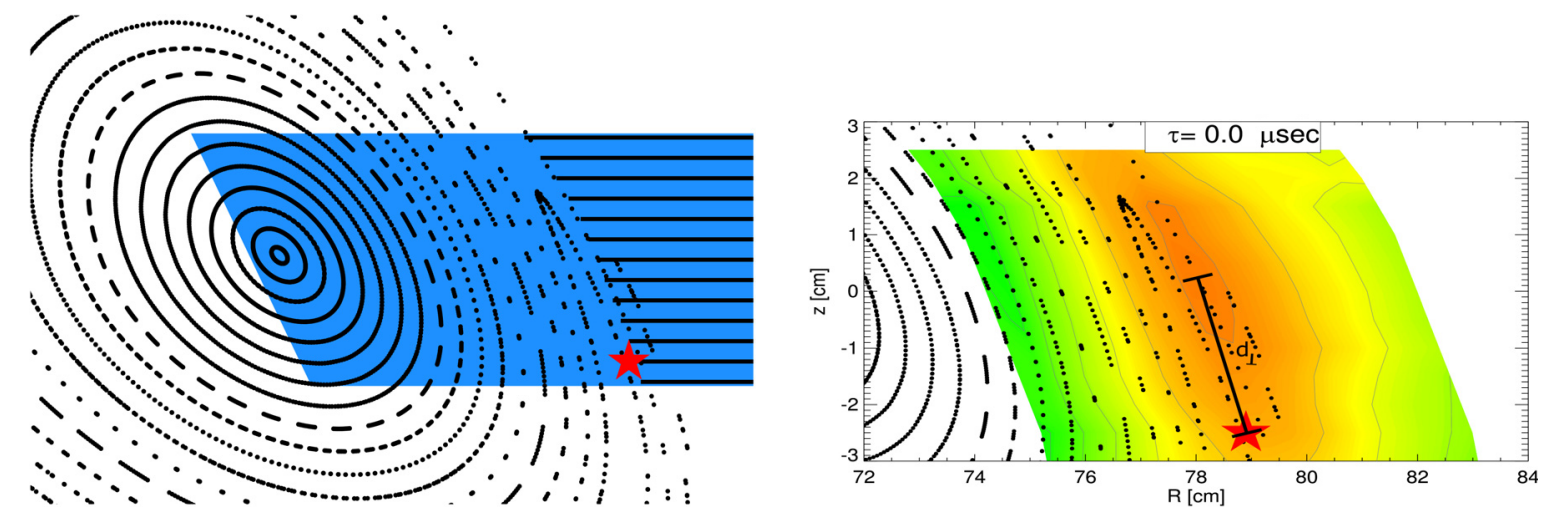

FIGURE 5. Left: Sketch of the poloidal probe array with 13 tips aligned to the flux surfaces. The red star marks the intersection with a field line starting at the reference probe. Right: Relative cross-correlation between reference probe and matrix in a poloidal plane.

The parallel velocity, determined from the cross-correlation of two probes on one field line is $v_{\|}=2.1 \times 10^{5} \mathrm{~m} / \mathrm{s}$. Theoretically, $v_{\|}$is expected to be in the range between $\mathrm{v}_{\mathrm{dia}} L_{\mathrm{c}} / d_{\perp}$ and the Alfvén velocity $v_{\mathrm{A}}$ [5]. For WEGA $v_{\mathrm{dia}}$ is some $\mathrm{km} / \mathrm{s}$, so the detected $v_{\|}$is close to its lower boundary and much smaller then $v_{\mathrm{A}} \sim 10^{6} \mathrm{~m} / \mathrm{s}$.

These experiments also show real three-dimensional character of turbulence in WEGA. The highest correlation has been found for a non-zero time lag of $\tau_{1} \sim 30 \mu \mathrm{s}$. This result corresponds to the picture of turbulence as being three-dimensional toroidally elongated filaments, so called 'blobs' [6]. The parallel expansion of the blob leads to a finite time lag of the correlation maximum.

In conclusion, it could be shown, that drift waves are the dominant instability mechanism driving turbulence in low field operation of the WEGA Stellarator.

The flexibility of WEGA can be used to study the impact of the magnetic field topology on plasma confinement. One issue is the study of turbulence and transport in the region of low rational magnetic islands. As described above the EFCC can be used to influence width and position of islands. A first result of Langmuir probe measurements in the region of islands can be seen in Fig. 6 where equilibrium density profiles from discharges at $B_{0}=0.5 \mathrm{~T}$ in $\mathrm{Ar}$ are plotted.

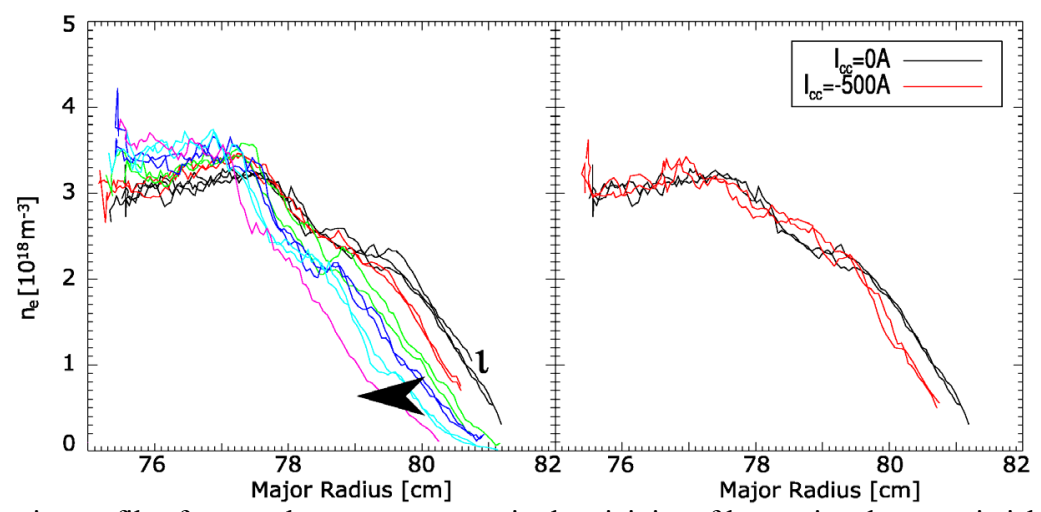

FIGURE 6. Left: Density profiles from probe measurements in the vicinity of low rational magnetic islands. A flattening observed at $\mathrm{t} / 2 \pi=1 / 4$ is shifted inwards by small variations of . Right: The flattening can be reduced by use of the EFCC.

Fig. 6 (left) shows profiles from a helical field scan where $\imath$ was varied by about $10 \%$. In all cases a flattening of profiles is detected in the vicinity of the $1 / 2 \pi=1 / 4$ resonance which is shifted from $R \sim 79 \mathrm{~cm}$ inward by about $1 \mathrm{~cm}$ during this scan. In order to heal this flattening the EFCC has been used at the maximum current of -500A where a strong decrease in island width has been observed in flux surface measurements. However, the inherent error field could not be fully compensated but the influence on the density profiles can be seen in Fig. 6 (right). The profile is getting much smoother at the resonance together with a steepening of the gradient outside the island. Based on these 
measurements, demonstrating the existence of magnetic islands and their accessibility with probe diagnostics, further studies on turbulence and transport in the vicinity of islands are currently being undertaken.

\title{
DIAGNOSTIC DEVELOPMENT
}

\begin{abstract}
HIBP
In collaboration with the Institute for Plasma Physics in Kharkov/Ukraine a Heavy Ion Beam Probe (HIBP) diagnostic is currently being tested on WEGA [7].

The detailed knowledge of the electric field structure and its fluctuations from the core to the edge of the plasma is fundamentally important for understanding of the plasma confinement phenomena. A Heavy Ion Beam Probe (HIBP) is the unique diagnostic, which is able to provide direct plasma electric potential measurements and its fluctuations from center to the periphery of the plasma column. The diagnostic is based on the injection of the singly charged ion beam across the confining magnetic field. Some probing particles are ionized in the plasma and produce mainly doubly ionized secondary ions. The information about plasma parameters can be obtained from their characteristics. So, plasma potential obtained by this diagnostics is the difference between primary and secondary beam energies. HIBP can be used in magnetic confinement fusion experiments to measure the electric potential $V_{\mathrm{p}}$, electron density $n_{\mathrm{e}}$ and electron temperature $T_{\mathrm{e}}$, poloidal magnetic field $B_{\vartheta}$ as well as their fluctuations with a high temporal $(1 \div 10 \mu \mathrm{s})$ and spatial $(0.5 \div 1 \mathrm{~cm})$ resolution. The diagnostic is optimized for a nominal magnetic field of $B_{0}=0.5 \mathrm{~T}$.

The HIBP that is currently under tests at WEGA uses $\mathrm{Na}^{+}$ions with an energy of $39 \mathrm{keV}$, a current up to $50 \mu \mathrm{A}$ and beam width $\sim 5 \mathrm{~mm}$. The spatial resolution of $5 \mathrm{~mm}$ can be obtained along the detector line (or radial scan). The position of the sample volume over the detector line is controlled by deflection plates voltage and covers a radial range of $0.4<\mathrm{r} / \mathrm{a}<1$. However, the plasma centre $(\mathrm{r} / \mathrm{a}=0)$ is not accessible due to geometrical limitations.

Potential measurement results indicate a positive plasma potential, using the vacuum vessel as the potential reference. This is in reasonable agreement with the data from the Langmuir probes.
\end{abstract}

\section{PREPARATIONS FOR WENDELSTEIN 7-X}

The WEGA stellarator is also used for tests of diagnostics that are foreseen for Wendelstein 7-X. Although, the plasma parameters of the core plasma are by orders of magnitude higher than in WEGA the plasma parameters in the plasma edge and the divertor region are comparable. Other diagnostics like the magnetic diagnostic could benefit from the long pulse operation of WEGA, which could be successfully shown for $2.45 \mathrm{GHz}$ ECR heating for $30 \mathrm{~min}$. In the following examples for the diagnostic development and the control system for Wendelstein 7-X are discussed.

\section{Coherence Imaging Spectrometer and Tomographic OES}

Current spectroscopic diagnostics under development on WEGA feature two systems, a coherence imaging spectrometer and a tomographic optical emission spectroscopy system for touch-free measurements of electron density and temperature.

Coherent Imaging Spectroscopy (CIS) has proven to be a very useful and flexible tool in plasma diagnostics [8]. The CIS diagnostic is very similar to a Fourier-transform spectrometer. After passing an interference filter, the light coming from the plasma is split into ordinary and extraordinary rays by bifringent crystals. It is then electrooptically modulated and the extraordinary ray is delayed by a determined phase. The ordinary and extraordinary rays interfere and the interferogram is recorded on a camera. The first three moments of the signal correspond to the intensity of the selected line, temperature of the emitting ions and the ion speed. By using different interference filters the species observed can be selected and by matching the delay crystals to the expected temperature range the sensitivity of the system can be further optimised.

Due to the low level of light emission the system used on WEGA captures images with a frame rate of $\sim 10 \mathrm{~Hz}$ from which plasma flow and temperature are derived [9]. Typically argon ion temperatures are $\sim 3 \mathrm{eV}$ having a 
maximum near the last closed flux surface. Argon plasmas typically rotate poloidally with a speed of around $1000 \mathrm{~m} / \mathrm{s}$ near the last closed flux surface. This has been independently measured with the coherence imaging spectrometer as well as high- resolution spectroscopy. It was found that the poloidal rotation of the ions consists of two major components - the $\mathrm{E} \times \mathrm{B}$-drift and the ion diamagnetic drift. The drift components are derived from combining Langmuir probe data with ion temperature measurements from spectroscopy. Combining the probe measurements, CIS as well as classical high-resolution spectroscopy allows not only determining the absolute value of the net poloidal rotation of the ions, but also the drift components.

The system is also used to study the impact of edge biasing on the net ion motion in WEGA. After installing the $28 \mathrm{GHz}$ gyrotron on WEGA the need for passive measurements of electron density and temperature rose as the plasma conditions became harsher for probe measurements. Hence, an additional optical emission diagnostic to tomographically derive electron density and temperature from well-known line intensity ratio technique is being constructed.

For W7-X it could be possible to use CIS in the divertor region where ion temperatures in the range of 1-100 eV are expected. The system is steady-state capable as is demonstrated on WEGA.

\section{Prototype of Control System}

For testing the new and complex control system of Wendelstein 7-X the WEGA experiment was chosen as a test bed. This concept phase will demonstrate the applicability of the segment-orientated control system at a running experiment including steady-state operation, interaction of all relevant components, real-time control, data acquisition and integrated data analysis. Furthermore, the safety concept will be implemented at WEGA and tested.

At the end of 2007 all components necessary for operation like the central control system, the power supplies, the cooling system will be installed and tested in autonomous operation. Starting from spring 2008 it will be possible operate WEGA with the integrated control system. For the future it is planned to integrate an increasing number of diagnostics to show the scalability.

\section{ACKNOWLEDGMENTS}

The authors gratefully acknowledge the work of D. Aßmus, N. Paschkowski and R. Gerhardt for their technical support.

\section{REFERENCES}

1. K. Horvath, J. Lingertat, M. Otte, and Friedrich Wagner, Plasma Phys. Control. Fusion 48 (2006) 315-323

2. R. Jaenicke et al., Nucl. Fusion 11 (1993) 687

3. J. Preinhalter and V. Kopeck'y, J. Plasma Phys. 10 (1973) 1

4. Y.Y. Podoba et al., Phys. Rev. Lett. 98255003 (2007)

5. N. Mahdizadeh et al, Plasma Phys. Control. Fusion 49, 1005 (2007)

6. S.J. Zweben and R.W. Gould, Nucl. Fusion 25, 171 (1985)

7. L. I. Krupnik et al., Fusion Science and Technology 50, (2006) 276-280

8. J.Howard, C.Michael, F.Glass and A.D. Cheetham, Review of Scientific Instrumensts 72 (2001) 888-897

9. J. Chung, R. König, J. Howard, M. Otte and T. Klinger, Plasma Phys. Control. Fusion 47 (2005) 919-940

10. A. Werner, Max-Planck-Institut für Plasmaphysik, Greifswald branch, Germany, private communication

11. J.M. Canik et al., Phys. Rev. Lett. 98, 085002 (2007) 